\title{
Generalizing Jouravski Formulas by Techniques from Differential Geometry
}

\author{
FRANCESCO DELL'ISOLA \\ GIUSEPPE C. RUTA \\ Università di Roma "La Sapienza," Facoltà di Ingegneria, via Eudossiana 18, I-00184 Roma, \\ Italy
}

(Received 5 December 1996; Final version 12 February 1997)

\begin{abstract}
We deal with the flexure (flexion inégale) of a Saint-Venant cylinder whose sections we call Bredtlike with variable thickness. We consider a family of sections $\mathcal{D}_{\varepsilon}$ whose thickness is scaled by a parameter $\varepsilon$. This scaling allows for the construction of an $\varepsilon$-one-parameter family of coordinate mappings from a fixed-plane domain $\mathcal{D}$ onto $\mathcal{D}_{\varepsilon}$. We represent the Helmholtz operator in $\mathcal{D}_{\varepsilon}$ in terms of a fixed system of coordinates in $\mathcal{D}$ and represent the shear stress field in what we call the Bredt basis field, which is not the natural basis associated with any coordinate system. Assuming that the shear stress admits a formal $\varepsilon$ power series expansion, we obtain a hierarchy of perturbation problems for its coefficients, finding the well-known Jouravski formula at the lowest iterative step and obtaining its generalization at higher steps-that is, when the section becomes thick. Similar results are obtained for the warping, the resultant shear stress, and the shear shape factors.
\end{abstract}

\section{GEOMETRY OF Bredt-like SECTIONS WITH VARIABLE THICKNESS}

We study here the plane Saint-Venant problem for shear stress in flexure for Bredt-like sections (dell'Isola and Ruta [1]). They are obtained by symmetrically thickening a plane curve $\mathcal{L}$ (middle line) along its Frenet normal with regularly varying thickness. The middle line is given as a function of its arc length $s$ :

$$
\mathcal{L}:=\left\{q \in \mathcal{P} \mid q-o=\mathbf{r}_{0}(s), \quad s \in[0, l]\right\}
$$

$o$ is any point in the plane $\mathcal{P}$ including the section, and $l$ is the length of $\mathcal{L}$. The Frenet orthonormal basis for the middle line is

$$
\mathbf{l}(s):=\frac{\partial \mathbf{r}_{0}(s)}{\partial s}=\mathbf{r}_{0, s}(s), \quad \mathbf{m}(s):=-* \frac{\partial \mathbf{r}_{0}(s)}{\partial s}=-* \mathbf{r}_{0, s}(s)
$$

* is the Hodge operator in $\mathcal{P}$ ( $\frac{\pi}{2}$ rotation about the positive orientation of $P$ ); the comma followed by a variable indicates a derivative with respect to that variable. The $\varepsilon$ Bredtlike section is given by

$$
\mathcal{D}_{\varepsilon}:=\left\{\mathbf{y} \in \mathcal{P} \mid \mathbf{y}-\mathbf{0}=\mathbf{r}(s, z)=\mathbf{r}_{0}(s)+z \varepsilon \delta(s) * \mathbf{l}(s), \quad s \in[0, l], z \in[-1,1]\right\} ;
$$


$z$ is the coordinate along $* \mathbf{l}(s), \delta(s)$ is the (suitably regular) function expressing half the thickness of the section, and $\varepsilon$ is a thickness perturbation parameter. That is, a Bredt-like section is the collection of all symmetrically $z$-shifted lines along $\mathbf{m}$ starting from $\mathcal{L}$, the shift being a regular function of the arc length $s$ of the middle line. We regard $s, z$ as rescaled coordinates over $\mathcal{D}_{\varepsilon}$ (Nayfeh [2]). The natural (covariant) basis associated with the $s, z$ coordinates is (Malvern [3])

$$
\begin{aligned}
& \mathbf{g}_{1}(s):=\frac{\partial \mathbf{r}}{\partial s}=[1-z \mathbf{K}(s) \varepsilon \delta(s)] \mathbf{l}(s)+z \varepsilon \delta_{, s}(s) * \mathbf{l}(s), \\
& \mathbf{g}_{2}(s):=\frac{\partial \mathbf{r}}{\partial z}=\varepsilon \delta(s) * \mathbf{l}(s)
\end{aligned}
$$

where $\kappa(s)$ stands for the curvature of the middle line. Henceforth, to lighten the notation and when there is no risk of confusion, we will drop the dependence of functions on coordinates $s, z$. The covariant components of the metric tensor are

$$
g_{11}=(1-z \kappa \varepsilon \delta)^{2}+\left(z \varepsilon \delta_{, s}\right)^{2}, \quad g_{12}=g_{21}=z \varepsilon^{2} \delta \delta_{, s}, \quad g_{22}=\varepsilon \delta^{2} .
$$

As a consequence, the determinant of the metric tensor is

$$
g=g_{11} g_{22}-\left(g_{11}\right)^{2}=(\varepsilon \delta)^{2}(1-z \kappa \varepsilon \delta)^{2}
$$

The basis dual to the natural one is obtained by the standard relation $\mathbf{g}^{i} \cdot \mathbf{g}_{j}=\delta_{j}^{i}$, where - stands for the usual scalar product in the vector space $\mathcal{V}$ of the translations of $\mathcal{P}$ :

$$
\mathbf{g}^{1}=\frac{\mathbf{l}}{1-z \kappa \varepsilon \delta}, \quad \mathbf{g}^{2}=-\frac{z \delta_{, s}}{\delta(1-z \kappa \varepsilon \delta)} \mathbf{l}+\frac{1}{\varepsilon \delta} * \mathbf{l} .
$$

As the section has variable thickness, the coordinate system $(s, z)$ is not, in general, orthogonal (it is on the middle line, by construction). For the purpose of this article, we need to calculate the Christoffel symbols (of second kind) associated with the coordinates $s, z$, according to $\left\{\begin{array}{c}j \\ k l\end{array}\right\}:=\mathbf{g}_{k, l} \cdot \mathbf{g}^{j}$ ([3]; dell'Isola and Rosa [4, 5]):

$$
\begin{array}{r}
\left\{\begin{array}{c}
1 \\
11
\end{array}\right\}=-\frac{z \varepsilon\left(2 \kappa \delta_{, s}+\kappa_{s,} \delta\right)}{1-z \kappa \varepsilon \delta}, \quad\left\{\begin{array}{c}
2 \\
11
\end{array}\right\}=\frac{z^{2} \varepsilon \delta_{s}\left(2 \kappa \delta_{s}+\kappa, s\right)}{\delta(1-\varepsilon z \kappa \delta)}+\frac{\kappa(1-\varepsilon z \kappa \delta)+z \delta_{, s s}}{\varepsilon \delta}, \\
\left\{\begin{array}{c}
1 \\
22
\end{array}\right\}=\left\{\begin{array}{c}
2 \\
22
\end{array}\right\}=0, \quad\left\{\begin{array}{c}
1 \\
21
\end{array}\right\}=\left\{\begin{array}{c}
1 \\
12
\end{array}\right\}=-\frac{\kappa \varepsilon \delta}{1-z \kappa \varepsilon \delta}, \quad\left\{\begin{array}{c}
2 \\
21
\end{array}\right\}=\left\{\begin{array}{c}
2 \\
12
\end{array}\right\}=\frac{\delta, s}{\delta(1-z \kappa \varepsilon \delta)} .
\end{array}
$$

Because of (1.3), the center $\mathbf{b}$ of area of the section is given by

$$
\mathbf{b}-\mathbf{o}=\frac{\int_{\mathcal{D}_{\varepsilon}}(\mathbf{y}-\mathbf{o})}{A_{\mathcal{D}_{\varepsilon}}}=\frac{\int_{0}^{l} \delta \mathbf{r}_{0}-\frac{\varepsilon^{2}}{3} \int_{0}^{l} \delta^{3} \kappa * \mathbf{l}}{\int_{0}^{l} \delta}:=\mathbf{b}_{0}-\varepsilon^{2} \mathbf{b}_{2},
$$

where $A_{\mathcal{D}_{\varepsilon}}$ is the area of the section. In all integrals, the measure of integration is omitted. 


\section{HELMHOLTZ OPERATOR WITH RESPECT TO THE BREDT BASIS}

The problem for the shear stress field $t$ in the flexure of a Saint-Venant cylinder is elliptic and defined over the $\varepsilon$ section as follows (Clebsch [6]; Fraeijs de Veubeke [7]):

$$
\begin{array}{rlrl}
\operatorname{div} \mathbf{t} & =Y \mathbf{k} \cdot[*(\mathbf{y}-\mathbf{b})] & \text { in } \quad \mathcal{D}_{\varepsilon}^{o}, \\
(\operatorname{curl} \mathbf{t}) \cdot \mathbf{e} & =2 G v \mathbf{k} \cdot(\mathbf{y}-\mathbf{0}) & \text { in } \quad \mathcal{D}_{\varepsilon}^{o}, \\
\mathbf{t} \cdot \mathbf{n} & =0 & \text { along } \quad & \partial \mathcal{D}_{\varepsilon}, \\
\oint_{\mathcal{L}=\partial \mathcal{R}} \mathbf{t} \cdot \mathbf{l} & =2 G v \mathbf{k} \cdot A_{\mathcal{R}}\left(\mathbf{b}_{\mathcal{R}}-\mathbf{o}\right) & \text { along } & \mathcal{L}(z=0) .
\end{array}
$$

In the former, $Y, G, v$ are the longitudinal (Young) elastic modulus, the tangential elastic modulus, and the Poisson ratio; $\mathbf{e}$ is the unit vector normal to the (positive) side of $\mathcal{P}$; $\mathbf{n}$ is the outer normal to $\partial \mathcal{D}_{\mathcal{E}} ; A_{\mathcal{R}}$ is the area of the inner Jordan region enclosed by $\mathcal{L}$ (if any); $\mathbf{b}_{\mathcal{R}}$ is its center of area; and $\mathbf{k}$ is the kinematic characteristic parameter of the flexure, representing the (linear) variation of the curvature of an initially straight axis of the cylinder. Equation (2.1) describes the local balance of contact force; equation (2.2) is a local compatibility condition, necessary and sufficient if the section is simply connected; equation (2.3) expresses the condition that the lateral surface of the cylinder be traction free; and equation (2.4) is a global compatibility condition to be used if the section has a connection higher than 1 .

It is well known that, in general, the problem has no closed-form analytical solution. In particular, in multiply-connected sections, the presence of sources of stress due to the lacunae makes the problem very complicated. It is clear that, for technical applications, approximate solutions can be useful. The most known technical formula for the shear stress in the case of flexure is that due to Jouravski (Feodosyev [8]; Gjelsvik [9]; Gavarini [10]) and is based on the integral counterpart of equation (2.1). The Jouravski formula provides a mean value for the shear stress component along $\mathcal{L}$, so supplying its accurate estimate when the section is thin. As far as we know, in the literature there is no approximate formula providing reliable results for thick sections; besides, for sections with a connection higher than 1, one finds formulas based on that of Jouravski, obtained by Volterra distorsions and applications of the principle of virtual power.

The $\varepsilon$ parameter in (1.3) describes sections with the same shape that grow thicker; we will obtain a hierarchy of perturbation problems in $\varepsilon$. The solution of each step of the hierarchy should provide generalized, Jouravski formulas approximating shear stress field of thick sections.

We need to write the Helmholtz operator present in equations (2.1) and (2.4) in the coordinate system $(s, z)$ in $\mathcal{D}$ and in terms of the thickness parameter $\varepsilon$. Besides that, to follow what is usually required for applications, we define the Bredt basis field $\mathcal{B}$ as:

$$
\mathcal{B}:(s, z) \mapsto \mathbf{I}(s), * \mathbf{l}(s) ;
$$

the components of its Lie bracket (Arnold [11]) in $(s, z)$ are

$$
[\mathbf{l}, * \mathbf{l}]_{1}=\frac{\kappa}{(1-z \kappa \varepsilon \delta)^{2}}, \quad[\mathbf{l}, * \mathbf{l}]_{2}=-\frac{z \kappa \delta_{s}}{\delta(1-z \kappa \varepsilon \delta)^{2}} ;
$$


that is, the Lie bracket of $\mathcal{B}$ is not, in general, the zero vector field. We may conclude that $\mathcal{B}$ is not, in general, the natural basis associated with any coordinate system. We do not represent the shear stress in the natural basis of the chosen coordinate system, but in the Bredt basis

$$
\mathbf{t}=t^{1} \mathbf{g}_{1}+t^{2} \mathbf{g}_{2}=t_{1} \mathbf{g}^{1}+t_{2} \mathbf{g}^{2}=t_{s} \mathbf{l}+t_{z} * \mathbf{l}
$$

Equation (2.7) facilitates finding relations between contravariant and Bredt components of the shear stress:

$$
t^{1}=\frac{t_{s}}{1-z \kappa \varepsilon \delta}, \quad t^{2}=\frac{1}{\varepsilon \delta} t_{z}-\frac{z \delta_{, s}}{\delta(1-z \kappa \varepsilon \delta)} t_{s} .
$$

The divergence operator is written as ([6], eq. 2.8)

$$
\operatorname{div} \mathbf{t}=(\nabla \mathbf{t})_{i}^{i}=t_{, s}^{1}+\left\{\begin{array}{c}
1 \\
j 1
\end{array}\right\} t^{j}+t_{, z}^{2}+\left\{\begin{array}{c}
2 \\
j 2
\end{array}\right\} t^{j}=\frac{t_{s, s}}{1-z \kappa \varepsilon \delta}-\frac{\kappa t_{z}}{1-z \kappa \varepsilon \delta}+\frac{t_{z, z}}{\varepsilon \delta}
$$

$\nabla$ being the spatial gradient operator. We remark that, when expressed in terms of $t_{s}, t_{z}$, the divergence operator is not affected by the variable thickness and the nonorthogonal coordinates. This is physically reasonable, as the divergence operator is linked more to the variation of the field along the middle line than to that along the thickness. This also intuitively accounts for the success of Jouravski heuristic deduction of his formula.

From equations (1.5) and (2.8), the covariant components of the shear stress may be found in terms of the Bredt components by $t_{i}=g_{i j} t^{j}$ as

$$
t_{1}=(1-z \kappa \varepsilon \delta) t_{s}+z \varepsilon \delta_{, s} t_{s}, \quad t_{2}=\varepsilon \delta t_{z}
$$

We need the covariant components of the shear stress to calculate the curl operator and express it in terms of the Bredt components. We have

$$
\begin{aligned}
(\text { curl }) \cdot \mathbf{e} & =\frac{1}{\sqrt{g}}\left(\nabla_{1} t_{2}-\nabla_{2} t_{1}\right)=\frac{t_{2, s}-\left\{\begin{array}{c}
j \\
12
\end{array}\right\} t_{j}-t_{1, z}+\left\{\begin{array}{c}
j \\
21
\end{array}\right\} t_{j}}{\varepsilon \delta(1-z \kappa \varepsilon \delta)} \\
& =\frac{t_{2, s}-t_{1, z}}{\varepsilon \delta(1-z \kappa \varepsilon \delta)}=\frac{t_{z, s}}{1-z \kappa \varepsilon \delta}-\frac{t_{s, z}}{\varepsilon \delta}+\frac{\kappa t_{s}}{1-z \kappa \varepsilon \delta}-\frac{z \delta_{, s} t_{z, z}}{\delta(1-z \kappa \varepsilon \delta)}
\end{aligned}
$$

We remark that equation (2.11) differs only in the fourth term from the expression one would obtain in the case of constant thickness. We also remark that this difference is exactly what one would expect in this case because the curl operator is linked with the variation of the field along the thickness. As a matter of fact, the different term we observe is proportional to the derivative of the $z$-component of the field with respect to $z$ and to the variation of the thickness along $\mathcal{L}$. We will also need the expression for the gradient of a scalar function $w$ of coordinates $s, z$, as we will look for the warping of the section. It is

$$
\nabla w=\frac{\partial w}{\partial s} \mathbf{g}^{1}+\frac{\partial w}{\partial z} \mathbf{g}^{2}=\left[\frac{\delta w_{, s}-z \delta_{, s} w_{, z}}{\delta(1-z \kappa \varepsilon \delta)}\right] \mathbf{I}+\frac{w_{, z}}{\varepsilon \delta} * \mathbf{l}
$$


equation (2.12) reduces to the expression of the gradient of a scalar function in an orthogonal coordinate system [11] if we let $\delta_{, s}=0$, as we would expect.

\section{FORMAL EXPANSIONS FOR THE SHEAR STRESS}

Since the thickness parameter describes a geometrical feature of the section, it is physically reasonable to let $\mathbf{t}$ be a function of $\varepsilon$ as well as of $s, z$. We propose the following formal series expansion for $\mathbf{t}[2]$ :

$$
\mathbf{t}(s, z ; \varepsilon)=\sum_{n=0}^{N} \varepsilon^{n} \mathbf{t}_{n}(s, z)+o\left(\varepsilon^{N}\right)
$$

where $o\left(\varepsilon^{N}\right)$ stands for terms of order higher than $\varepsilon^{N}$. Similar formal expansions are also supposed to be valid for both components of the shear stress field with respect to the Bredt basis.

Equations (2.9), (2.11), and (3.1), when substituted into (2.1) through (2.4), lead to the following hierarchy of perturbation problems:

$$
\frac{\sum_{n=0}^{N}\left\{\varepsilon^{n+1} \delta\left[t_{s n, s}-\kappa\left(t_{z n}+z t_{z n, z}\right)\right]+\varepsilon^{n} t_{z n, z}\right\}}{\varepsilon \delta(1-z \kappa \varepsilon \delta)}=Y \mathbf{k} \cdot\left(* \overline{\mathbf{r}}_{0}-\varepsilon z \delta \mathbf{I}+\varepsilon^{2} \mathbf{b}_{2}\right),
$$

$\frac{\sum_{n=0}^{N}\left\{\varepsilon^{n+1}\left[\delta t_{z n, s}+\kappa \delta\left(t_{s n}+z t_{s n, z}\right)-z \delta_{, s} t_{z n, z}\right]-\varepsilon^{n} t_{s n, z}\right\}}{\varepsilon \delta(1-z \kappa \varepsilon \delta)}=2 G v \mathbf{k} \cdot\left(\mathbf{r}_{0}+\varepsilon z \delta * \mathbf{l}\right)$,

$$
\begin{aligned}
\sum_{n=0}^{N} \varepsilon^{n} \mathbf{t} \cdot \mathbf{n} & =0, \\
\sum_{n=0}^{N} \oint_{z=0} \varepsilon^{n} t_{s n} & =2 G v \mathbf{k} \cdot A_{\mathcal{R}}\left(\mathbf{b}_{\mathcal{R}}-\mathbf{o}\right) .
\end{aligned}
$$

In equations (3.2) through (3.5), we have dropped terms of order higher than $\varepsilon^{N}$; in equation (3.2), $\overline{\mathbf{r}}_{0}:=\mathbf{r}_{0}-\mathbf{b}_{0}$; in equation (3.4), $\mathbf{n}$ is the outer unit normal vector.

If the section has double connection, we will call it closed, referring to its middle line, which is homotopic to a circumference. In this case, two different connected elements compose the boundary of the section. The outer vector field normal to the boundary is given by

$$
\left.\mathbf{n}\right|_{z=-1}=-\varepsilon \delta_{, s} \mathbf{l}-(1+\varepsilon \kappa \delta) * \mathbf{l},\left.\quad \mathbf{n}\right|_{z=1}=-\varepsilon \delta_{, s} \mathbf{l}+(1-\varepsilon \kappa \delta) * \mathbf{l},
$$


so that the boundary conditions for closed sections are, dropping terms $o\left(\varepsilon^{N}\right)$,

$$
\begin{gathered}
\sum_{n=0}^{N}\left|\varepsilon^{n+1}\left(\delta_{, s} t_{s n}+\kappa \delta t_{z n}\right)+\varepsilon^{n} t_{z n}\right|_{z=-1}=0 \\
\sum_{n=0}^{N}\left|-\varepsilon^{n+1}\left(\delta_{s} t_{s n}+\kappa \delta t_{z n}\right)+\varepsilon^{n} t_{z n}\right|_{z=1}=0 .
\end{gathered}
$$

If the section is simply connected, we will call it open, always referring to its middle line, now homotopic to a segment. The boundary is composed by a unique connected element, divided into four regular components. The outer vector field normal to the boundary is represented by

$$
\begin{aligned}
\left.\mathbf{n}\right|_{s=0}=-\mathbf{l}, & \left.\mathbf{n}\right|_{z=-1}=-\varepsilon \delta_{s} \mathbf{l}-(1+\varepsilon \kappa \delta) * \mathbf{l}, \\
\left.\mathbf{n}\right|_{s=l}=\mathbf{l}, & \left.\mathbf{n}\right|_{z=1}=-\varepsilon \delta_{s} \mathbf{l}+(1-\varepsilon \kappa \delta) * \mathbf{l},
\end{aligned}
$$

so that boundary conditions for open sections are, always dropping terms $o\left(\varepsilon^{N}\right)$,

$$
\begin{array}{r}
\sum_{n=0}^{N}\left|\varepsilon^{n} t_{s n}\right|_{s=0}=0 \\
\sum_{n=0}^{N}\left|\varepsilon^{n+1}\left(\delta_{, s} t_{s n}+\kappa \delta t_{z n}\right)+\varepsilon^{n} t_{z n}\right|_{z=-1}=0 \\
\sum_{n=0}^{N}\left|\varepsilon^{n} t_{s n}\right|_{s=l}=0 \\
\sum_{n=0}^{N}\left|-\varepsilon^{n+1}\left(\delta_{s} t_{s n}+\kappa \delta t_{z n}\right)+\varepsilon^{n} t_{z n}\right|_{z=1}=0 .
\end{array}
$$

In the next sections, we will look for the solution of the hierarchy of perturbation problems (3.2) through (3.5), with the appropriate substitution of the boundary conditions (3.7), (3.8), and (3.11) through (3.14), both for closed and open sections.

\section{GENERALIZED JOURAVSKI FORMULAS}

In the following, we deduce some generalizations of the Jouravski formula and study the main features of the structure of the aforementioned hierarchy of problems.

First, we remark that each step of the procedure can be solved within an unknown function of the coordinate $s$, which is determined only by solving the first equation of the next step. This is a very well-known feature of some perturbation series [2].

Second, both for closed and open sections the structure of the perturbation problem is the same except for two different boundary conditions. Anyway, in the case of open sections, we cannot force the formal series (3.1) to fulfill four independent boundary 
conditions. This is another well-known phenomenon of perturbation procedures, in the presence of a boundary layer (in this case, in a neighborhood of the "short" sides of the section $[1,2,4,5])$. We should provide two different expansions, called outer and inner [2], and then match them. But for the aim of this article, we will content ourselves with the outer expansion, which is valid outside the region of the boundary layer.

Third, the aforementioned features for the series (3.1) remain unchanged if we are to study the torsion of a Saint-Venant cylinder with Bredt-like sections, as defined in Section 1. In fact, if we modify the right-hand sides of equations (2.1) through (2.4) as follows,

$$
\begin{aligned}
& \operatorname{div} \mathbf{t}=0 \quad \text { in } \quad \mathcal{D}_{\varepsilon}^{o}, \\
& \text { (curlt) } \cdot \mathbf{e}=2 G \tau \quad \text { in } \quad \mathcal{D}_{\varepsilon}^{o}, \\
& \mathbf{t} \cdot \mathbf{n}=0 \quad \text { along } \quad \partial \mathcal{D}_{\varepsilon}, \\
& \oint_{\mathcal{L}=\partial \mathcal{R}} \mathbf{t} \cdot \mathbf{l}=2 G \tau A_{\mathcal{R}} \quad \text { along } \quad \mathcal{L}(z=0),
\end{aligned}
$$

we have a torsion problem $[6,7]$ in which $\tau$, the kinematic parameter, stands for the unit angle of twist. If we postulate for the shear stress field the formal series expansion (3.1), we obtain a hierarchy of perturbation problems that can be easily solved, thus obtaining again some of the results presented in $[1,4,5]$.

Now, starting from open sections (simply connected), we will solve the hierarchy of perturbation problems for the flexure, given by equations (3.2) and (3.3), with equation (3.4) replaced by equations (3.11) through (3.14). We obtain

$$
\begin{aligned}
\mathbf{t}_{0} & =\left[Y \mathbf{k} \cdot \frac{\int_{0}^{s}\left(\delta * \overline{\mathbf{r}}_{0}\right)}{\delta}\right] \mathbf{l}:=t_{s 0} \mathbf{l}, \\
\mathbf{t}_{1} & =z\left\{\delta\left[\kappa t_{s 0}-2 G v \mathbf{k} \cdot \mathbf{r}_{0}\right] \mathbf{l}+\left(\delta_{s} t_{s 0}\right) * \mathbf{l}\right\}, \\
\mathbf{t}_{2} & =\left\{\frac{z^{2}}{2}\left[\delta\left(\delta_{s s} t_{s 0}\right)_{, s}-\delta_{s}^{2} t_{s 0}\right]+z^{2} \delta^{2}\left[\kappa^{2} t_{s 0}-G \mathbf{k} \cdot\left(\kappa \mathbf{r}_{0}+* \mathbf{l}\right)\right]+\bar{t}_{s 2}\right\} \mathbf{l} \\
& +\left\{\delta \delta_{s s}\left[\left(z^{2}+1\right) \kappa t_{s 0}+\left(z^{2}-3\right) G v \mathbf{k} \cdot \mathbf{r}_{0}\right]\right. \\
& \left.+\left(1-z^{2}\right) \delta^{2}\left[\frac{\kappa_{s} t_{s 0}}{2}+\mathbf{k} \cdot\left(G \mathbf{l}+Y \kappa * \overline{\mathbf{r}}_{0}\right)\right]\right\} * \mathbf{l} .
\end{aligned}
$$

Equation (4.5) is the well-known Jouravski formula [8-10]: in fact,

1. the integral on the right-hand side represents the lowest term of the first moment of area of the section;

2. from the general theory of Saint-Venant problem $[6,7]$, it is known that

$$
\mathbf{k}=-\frac{\mathbf{J}^{-1} * \mathbf{q}}{Y}, \quad \mathbf{q}:=\int_{\mathcal{D}_{\mathfrak{e}}} \mathbf{t}, \quad \mathbf{J}:=\int_{\mathcal{D}_{\mathfrak{R}}}(* \mathbf{r} \otimes * \mathbf{r}) .
$$

In equation (4.8), $\otimes$ is the tensor product between vectors of $\mathcal{V}$, defined by $(\mathbf{a} \otimes \mathbf{b}) \mathbf{c}:=(\mathbf{a} \cdot \mathbf{c}) \mathbf{b} ; \mathbf{q}$ is the resultant shear stress, and $\mathbf{J}$ is a tensor of inertia of the section; 
3. the Jouravski formula is usually given in the literature in components with respect to the principal axes of inertia of the section and in terms of the resultant shear stress $q$; it is easy to recognize it by substituting equation $(4.8)_{1}$ into equation (4.5).

We obtained the Jouravski formula as the solution of the first step of our perturbation procedure; because of the physical interpretation of the perturbation parameter, this corresponds to sections that in the literature are referred to as thin.

Equation (4.6) is the first generalization of the Jouravski formula for sections that are getting thicker, according to the interpretation of the successive powers of the perturbation parameter. We remark that the component orthogonal to I of this field may be found in the literature [8-10]: it is obtained, on the basis of the Jouravski formula, by solving the local equation of compatibility (3.3). It is said, on heuristic grounds, that this field is of little relevance for thin sections, if compared with that given by the Jouravski formula; in our perturbation approach, this result appears clearly as a higher order effect. As far as we know, the component of $t_{1}$ parallel to $l$ is not given in the literature.

We remark also that

1. equation (4.5) satisfies all boundary conditions (3.11) through (3.14);

2. equation (4.6) satisfies the boundary conditions (3.11) and (3.13) at the short sides of the section as a mean over the thickness;

3. starting from the third step of the procedure, we cannot satisfy boundary conditions at the short sides of the section because of an edge effect near the short elements of the boundary [2].

The fact that the first two terms of the outer $\varepsilon$ expansion of the shear stress field in flexure satisfy, at least in the mean, all boundary conditions is of great importance. In fact, when we study torsion, even at the lowest order of the expansion, it is not possible to satisfy all boundary conditions, and an edge boundary layer effect, named after Kelvin, arises [5]. In the case of flexure, this does not happen, as we will show also in the next section: the first two terms of the $\varepsilon$ expansion for the resultant shear stress, which we will calculate using equations (4.5), (4.6), and (4.8) 2 , coincide with those obtained using equation $(4.8)_{1}$. Thus, even if we have considered only the outer expansion of the shear stress, we obtain exactly the global effects predicted by the Saint-Venant theory. We may so conclude that in the case of flexure, the edge boundary layer effect is absent at the lowest two orders of our perturbation procedure.

In equation (4.7), $\bar{t}_{s 2}$ is a function of $s$ only, which is given implicitly by solving the local equation of balance at the following step, together with the boundary conditions on the long elements of the border.

The solutions of the first three steps of the hierarchy of perturbation problems for sections with double connection (closed), given by equations (3.2) through (3.5), with 
the substitution of the boundary conditions (3.7) and (3.8) into (3.4), are

$$
\begin{aligned}
\mathbf{t}_{0} & =\left\{\frac{Y \mathbf{k}}{\delta} \cdot\left[\int_{0}^{s}\left(\delta * \overline{\mathbf{r}}_{0}\right)-\frac{\oint_{\mathcal{L}} \frac{\int_{0}^{s}\left(\delta * \overline{\mathbf{r}}_{0}\right)}{\delta}}{\oint_{\mathcal{L}} \frac{1}{\delta}}\right]+\frac{2 G A_{\mathcal{R}} v \mathbf{k} \cdot\left(\mathbf{b}_{\mathcal{R}}-\mathbf{o}\right)}{\delta \oint_{\mathcal{L}} \frac{1}{\delta}}\right\} \mathbf{l}:=t_{s 0} \mathbf{l}, \\
\mathbf{t}_{1} & =z\left\{\delta\left[\kappa t_{s 0}-2 G v \mathbf{k} \cdot \mathbf{r}_{0}\right] \mathbf{l}+\left(\delta_{s s} t_{s 0}\right) * \mathbf{l}\right\} \\
\mathbf{t}_{2} & =\left\{\frac{z^{2}}{2}\left[\delta\left(\delta_{s} t_{s 0}\right)_{s}-\delta_{s}^{2} t_{s 0}\right]+z^{2} \delta^{2}\left[\kappa^{2} t_{s 0}-G v \mathbf{k} \cdot\left(\kappa \mathbf{r}_{0}+* \mathbf{l}\right)\right]+\bar{t}_{s 2}\right\} \mathbf{l} \\
& +\left\{\delta \delta_{s}\left[\left(z^{2}+1\right) \kappa t_{s 0}+\left(z^{2}-3\right) G v \mathbf{k} \cdot \mathbf{r}_{0}\right]\right. \\
& \left.+\left(1-z^{2}\right) \delta^{2}\left[\frac{\kappa_{s} t_{s 0}}{2}+\mathbf{k} \cdot\left(G \mathbf{l}+Y \kappa * \overline{\mathbf{r}}_{0}\right)\right]\right\} * \mathbf{l} .
\end{aligned}
$$

Equation (4.9) is the Jouravski formula for thin, doubly connected sections, but in the literature it is not usually written in that form. In fact, in technical textbooks [8-10], it is said that, to find the (dominant component of the) shear stress for these sections, one should consider them as indetermined structures. One should so imagine to produce a Volterra distorsion along the middle line and to put there an unknown shear stress flow determined by the principle of virtual power. We instead obtain equation (4.9) simply by solving the equations of the first step of the perturbation hierarchy, in a direct and rational way, only on the basis of equation (3.1). In the literature, the second addend of $t_{s 0}$ is usually called torsion in the section as a whole because it has the same form as the Bredt field in the torsion of a thin, closed section [1, 4, 5, 8-10]; on the basis of our procedure, this similarity is more clear, as both come from the integral condition of compatibility, equations (3.5) and (4.4) $[1,4,5]$.

We remark that the generalized formulas obtained at higher steps have exactly the same form as those obtained for open sections (equations (4.5) through (4.7)). This is to be expected, as the structure of the hierarchy is the same in both cases; the only difference is the different form of $t_{s 0}$ caused by the use of the integral compatibility equation (3.5).

\section{APPLICATIONS AND CONCLUSIONS}

As a first application of the results found solving the initial steps of the hierarchy of perturbation problems (3.2) through (3.5), we will calculate the warping $w$ of the section. We have from the general theory $[6,7]$ neglecting contributions due to rigid body motion,

$$
\nabla w=\frac{\mathbf{t}}{G}+v\{[*(\mathbf{b}-\mathbf{o})] \otimes \mathbf{r}-\operatorname{sym}(\mathbf{r} \otimes * \mathbf{r})\} \mathbf{k},
$$

where $\operatorname{sym}(\mathbf{a} \otimes \mathbf{b})$ stands for the symmetric part of the indicated tensor.

We postulate a formal $\varepsilon$ power series expansion also for $w$ :

$$
w(s, z ; \varepsilon)=\sum_{n=0}^{N} \varepsilon^{n} w_{n}(s, z) .
$$


By means of equation (2.11), we substitute equation (5.2) into equation (5.1) and obtain the following hierarchy of perturbation problems, in which terms $o\left(\varepsilon^{N}\right)$ have been neglected:

$$
\begin{aligned}
& \sum_{n=0}^{N}\left[\varepsilon^{n}\left(\delta_{n, s}-z \delta_{, s} w_{n, z}\right) \mathbf{l}+\left(\varepsilon^{n}-z \kappa \delta \varepsilon^{n+1}\right) w_{n, z} * \mathbf{l}\right]=\frac{\varepsilon \delta-z \kappa \delta^{2} \varepsilon^{2}}{G}\left\{\sum_{n=0}^{N}\left(\varepsilon^{n} t_{s n} \mathbf{l}+\varepsilon^{n} t_{z n} * \mathbf{l}\right)\right. \\
& -v\left\{\operatorname{sym}\left(\mathbf{r}_{0} \otimes * \mathbf{r}_{0}\right)-* \mathbf{b}_{0} \otimes \mathbf{r}_{0}+\varepsilon z \delta\left[\operatorname{sym}\left(* \mathbf{l} \otimes * \mathbf{r}_{0}-\mathbf{r}_{0} \otimes \mathbf{l}\right)-* \mathbf{b}_{0} \otimes \mathbf{l}\right]\right. \\
& \left.+\varepsilon^{2}\left[z^{2} \delta^{2} \operatorname{sym}\left(* \mathbf{l} \otimes \mathbf{l}-* \mathbf{b}_{2} \otimes \mathbf{r}_{0}\right]+\varepsilon^{3} z \delta * \mathbf{b}_{2} \otimes * \mathbf{l}\right\} \mathbf{k}\right\}
\end{aligned}
$$

The structure of the hierarchy decouples a system of partial differential equations into a system of ordinary differential equations that is easier to solve. Besides that, we see from equation (5.3) that the right-hand side consists of

1. a first addend that contains the coefficients of the formal expansion of the shear stress field; we have seen from equations (4.5) through (4.7) and (4.9) through (4.11) that the structure of these coefficients is given in terms of the coefficient $t_{s 0}$ and is the same both for open and closed sections;

2. a second addend that is the same both for open and closed sections.

This means that the structure of the $w_{n}$ will be the same both for open and closed sections, with the only exception of $w_{0}$, influenced by the different form of $t_{s 0}$, given by equations (4.5) and (4.9). In fact, we have

$$
\begin{aligned}
& w_{0}=\frac{t_{s 0}}{G}-v \mathbf{k} \cdot \int_{0}^{s}\left[\operatorname{sym}\left(\mathbf{r}_{0} \otimes * \mathbf{r}_{0}\right)-* \mathbf{b}_{0} \otimes \mathbf{r}_{0}\right] \mathbf{l}, \\
& w_{1}=-v z \delta \mathbf{k} \cdot\left\{2 \int_{0}^{s} \mathbf{r}_{0}+\left[\operatorname{sym}\left(\mathbf{r}_{0} \otimes * \mathbf{r}_{0}\right)-\mathbf{r}_{0} \otimes * \mathbf{b}_{0}\right] * \mathbf{l}\right\}
\end{aligned}
$$

both for open and closed sections. Equation (5.5), so far as we know, is not found in the literature.

As a second application of our method, we will calculate the resultant shear stress over the section. From the general theory of the Saint-Venant problem [6, 7], $\mathbf{q}=Y * \mathbf{J k}$, with $\mathbf{q}$ and $\mathbf{J}$ given by equation (4.8). As the position vector field $\mathbf{r}$ is given by (1.3),

$$
\mathbf{J}=\varepsilon\left[2 \int_{0}^{l} \delta\left(* \mathbf{r}_{0} \otimes * \mathbf{r}_{0}\right)\right]-\varepsilon^{3}\left[2 \int_{0}^{l} z \kappa \delta^{2}\left(\mathbf{l} \otimes \mathbf{I}+\mathbf{l} \otimes * \mathbf{r}_{0}+* \mathbf{r}_{0} \otimes \mathbf{l}\right)\right]:=\varepsilon \mathbf{J}_{1}+\varepsilon^{3} \mathbf{J}_{3}
$$


We postulate that the resultant shear stress also admits a formal $\varepsilon$ power series expansion:

$$
\begin{aligned}
\mathbf{q}(\varepsilon)=\sum_{n=0}^{N} \varepsilon^{n} \mathbf{q}_{n}+o\left(\varepsilon^{N}\right) & =\int_{\mathcal{D}} \sum_{n=0}^{N} \sqrt{g} \varepsilon^{n} \mathbf{t}_{n}+o\left(\varepsilon^{N}\right) \\
& =Y *\left(\varepsilon \mathbf{J}_{1}+\varepsilon^{3} \mathbf{J}_{3}\right) \mathbf{k}
\end{aligned}
$$

the second line of equation (5.7) is implied by equation (5.6).

If we calculate the resultant shear stress according to the first line of equation (5.7) (i.e., in terms of the fields $\mathbf{t}_{0}, \mathbf{t}_{1}$ ) we obtain, both for open and closed sections,

$$
\begin{aligned}
& \mathbf{q}_{0}=\mathbf{0}, \\
& \mathbf{q}_{1}=Y * \mathbf{J}_{1} \mathbf{k}, \\
& \mathbf{q}_{2}=\mathbf{0} .
\end{aligned}
$$

Equation (5.8) implies that the shear stress resultant is zero when the thickness vanishes, which has a clear physical interpretation. Equation (5.9) shows that the resultant of the first two terms of the outer expansions for the shear stress is exactly that given by the second line of equation (5.7). This result is used in the literature, as in technical textbooks Jouravski shear stress field is a mean obtained starting from what we have called $\mathbf{q}_{1}$. Equation (5.10) follows from the symmetric construction of the section; such a result is sometimes implicitly assumed in the literature because it is said that the shear stress orthogonal to that of Jouravski is of little relevance, which means that its resultant will appear at least starting from order three in $\varepsilon$.

We remark that we obtain equations (5.8) through (5.10) both for open and closed sections. That is, even if we have found only the outer expansion for the shear stress field (valid outside the boundary layer), it provides exact resultants. This was to be expected, as we remarked earlier that equations (4.5) and (4.6) satisfy, at least in the mean, all boundary conditions - that is, the boundary layer effect is of higher order, and in flexure a Kelvin-type effect [5] is absent.

As a last application of our perturbation method, we will calculate the shear shape factor operator, which is a symmetric tensor $\mathbf{K}$ defined by an equivalence in the mechanical power spent by the shear stress and the resultant shear stress [8-10]:

$$
\frac{1}{2} \mathbf{q} \cdot \frac{\mathbf{K q}}{G A_{\mathcal{D}_{\mathfrak{e}}}}=\frac{1}{2 G} \int_{\mathcal{D}_{\mathfrak{\varepsilon}}}(\mathbf{t} \cdot \mathbf{t}) \Rightarrow \mathbf{q} \cdot \mathbf{K q}=A_{\mathcal{D}_{\mathfrak{\varepsilon}}} \int_{\mathcal{D}_{\mathfrak{\varepsilon}}}(\mathbf{t} \cdot \mathbf{t}) .
$$

The area $A_{\mathcal{D}_{\mathrm{\varepsilon}}}$ is given by the integration of $\sqrt{g}$ see equation (1.6) over the fixed domain $\mathcal{D}$.

We postulate for $\mathbf{K}$ a formal $\varepsilon$ power series expansion:

$$
\mathbf{K}(\varepsilon)=\sum_{n=0}^{N} \varepsilon^{n} \mathbf{K}_{n}+o\left(\varepsilon^{n}\right)
$$


Substituting the formal expansions for $\mathbf{K}$ (equation (5.12)), $\mathbf{q}$ (equation (5.7)), and $\mathbf{t}$ (equation (3.1)) into the definition (5.11), we obtain another perturbation hierarchy for the coefficients $\mathbf{K}_{n}$ :

$$
\sum_{n=0}^{N}\left(\varepsilon^{n} \mathbf{q}_{n} \cdot \varepsilon^{n} \mathbf{K}_{n} \varepsilon^{n} \mathbf{q}_{n}\right)=2 A_{\mathcal{D}_{\varepsilon}} \int_{-1}^{1} \int_{0}^{l} \sum_{n=0}^{N}\left(\varepsilon \delta-\varepsilon^{2} z \kappa \delta\right)\left(\varepsilon^{n} \mathbf{t}_{n} \cdot \varepsilon^{n} \mathbf{t}_{n}\right)
$$

where we have dropped terms $o\left(\varepsilon^{N}\right)$. Using results for $\mathbf{q}_{n}$ (equations (5.8) through (5.10)), we obtain

$$
\begin{aligned}
& \mathbf{q}_{1} \cdot \mathbf{K}_{0} \mathbf{q}_{1}=\left(\int_{0}^{l} \delta\right) \int_{-1}^{1} \int_{0}^{l} \delta \mathbf{t}_{0} \cdot \mathbf{t}_{0} \\
& \mathbf{q}_{1} \cdot \mathbf{K}_{1} \mathbf{q}_{1}=\left(\int_{0}^{l} \delta\right) \int_{-1}^{1} \int_{0}^{l}\left(2 \delta \mathbf{t}_{0} \cdot \mathbf{t}_{1}-z \kappa \delta^{2} \mathbf{t}_{0} \cdot \mathbf{t}_{0}\right)=0
\end{aligned}
$$

equation (5.15) is a direct consequence of equations (4.5) and (4.6), as well as (4.9) and (4.10). We remark that $\mathbf{K}_{1}$ vanishes due to the symmetry of the section and the linearity of the integrand in $z$.

It is very easy and meaningful to obtain the explicit expression of the coefficient $\mathbf{K}_{\mathbf{0}}$ in the case of open (simply connected) sections. Using the expressions for $t_{0}$, given by equation (4.5), we obtain, after some lengthy but easy calculation,

$$
\mathbf{K}_{0}=-2\left(\int_{0}^{l} \delta\right) * \mathbf{J}_{1}^{-1}\left[\int_{0}^{l} \frac{\left(\int_{0}^{s} \delta * \overline{\mathbf{b}}_{0}\right) \otimes\left(\int_{0}^{s} \delta * \overline{\mathbf{b}}_{0}\right)}{\delta}\right] \mathbf{J}_{1}^{-1} * .
$$

It is easy to prove that, if we chose an orthonormal basis whose elements are parallel to the principal axes of inertia of the section, as it is usually done in the textbooks, we obtain the approximate technical result for the component $K_{11}$ of the shear shape factor tensor [8-10]. As far as we know, only in [7] both components $K_{11}$ and $K_{22}$ of $\mathbf{K}_{0}$ are given, but it is not said clearly that the shear shape factor is a tensor, nor is its general expression (5.16) given.

In our opinion, the perturbation procedure involving the Helmholtz operator given by equations (2.1) through (2.4) is more efficient than those used in [1, 4, 5]. Indeed, it allows for

1. the treatment of the flexure, which is the most complicated of the four cases of simple deformation of a Saint-Venant cylinder, requiring a significantly reduced amount of calculation;

2. the immediate deduction of nearly all results obtained in $[1,4,5]$.

The problem to regularize the approximation sequence we obtain and to give convergence arguments remains open; this should be possible by following the ideas given in Wheeler and Horgan [12]. 


\section{REFERENCES}

[1] dell'Isola, F. and Ruta, G. C.: Outlooks in Saint-Venant theory I: Formal expansions for torsion of Bredt-like sections. Archives of Mechanics, 46(6), 1005-1027 (1994).

[2] Nayfeh, A. H.: Perturbation Methods, John Wiley, New York, 1973.

[3] Malvern, L. E.: Introduction to the Mechanics of a Continuous Medium, Prentice Hall, Englewood Cliffs, NJ, 1969.

[4] dell'Isola, F. and Rosa, L.: Outlooks in Saint-Venant theory II: Torsional rigidity, shear stress, "and all that" in the torsion of cylinders with sections of variable thickness. Archives of Mechanics, 48(4), 753-763 (1996).

[5] dell'Isola, F. and Rosa, L.: An extension of Kelvin and Bredt formulas. Mathematics and Mechanics of Solids, 1(2), 243-250 (1996).

[6] Clebsch, A.: Théorie de l'élasticité des corps solides (Traduite par MM. Barré de Saint-Venant et Flamant, avec des Notes étendues de M. Barré de Saint-Venant), Dunod, Paris, 1883. [Reprinted by Johnson Reprint Corporation, New York, 1966]

[7] Fraeijs de Veubeke, B. M.: A Course in Elasticity, Springer-Verlag, New York, 1979.

[8] Feodosyev, V. I.: Soprotivlenie Materialov, MIR, Moskwa, 1968. [Italian translation: Resistenza dei materiali, Editori Riuniti, Roma, 1977]

[9] Gjelsvik, A.: The Theory of Thin Walled Bars, John Wiley, New York, 1981.

[10] Gavarini, C.: Lezioni di Scienza delle Costruzioni, 3rd ed., ESA, Milano, Italy, 1996.

[11] Arnold, V. I.: Matematiceskie metody klassiceskoj mechaniki, MIR, Moskwa, 1979. [Italian translation: Metodi matematici della meccanica classica, Editori Riuniti, Roma, 1992]

[12] Wheeler, L. and Horgan, C. O.: Upper and lower bounds for the shear stress in the Saint-Venant theory of flexure. Journal of Elasticity, 6(4), 383-403 (1976). 